\title{
Using the World Wide Web to Connect Research and Professional Practice: Towards Evidence-Based Practice
}

\author{
Daniel L. Moody \\ Charles University \\ Prague, Czech Republic \\ moody@ksint.ms.mff.cuni.cz \\ Monash University \\ Melbourne, Australia
}

\section{Abstract}

In most professional (applied) disciplines, research findings take a long time to filter into practice, if they ever do at all. The result of this is under-utilisation of research results and sub-optimal practices. There are a number of reasons for the lack of knowledge transfer. On the "demand side", people working in professional practice have little time available to keep up with the latest research in their field. In addition, the volume of research published each year means that the average practitioner would not have time to read all the research articles in their area of interest even if they devoted all their time to it. From the "supply side", academic research is primarily focused on the production rather than distribution of knowledge. While they have highly developed mechanisms for transferring knowledge among themselves, there is little investment in the distribution of research results beyond research communities. The World Wide Web provides a potential solution to this problem, as it provides a global information infrastructure for connecting those who produce knowledge (researchers) and those who need to apply this knowledge (practitioners). This paper describes two projects which use the World Wide Web to make research results directly available to support decision making in the workplace. The first is a successful knowledge management project in a health department which provides medical staff with on-line access to the latest medical research at the point of care. The second is a project currently in progress to implement a similar system to support decision making in IS practice. Finally, we draw some general lessons about how to improve transfers of knowledge from research and practice, which could be applied in any discipline.

Keywords: knowledge management, evidence-based medicine (EBM), World Wide Web (WWW), IS research, IS practice, education, decision support system (DSS), web-based development

\section{Introduction}

\section{The Problem Addressed}

There is an enormous amount of new knowledge generated every year as a result of academic research. To make a practical difference, this knowledge needs to

Material published as part of this journal, either on-line or in print, is copyrighted by the publisher of Informing Science. Permission to make digital or paper copy of part or all of these works for personal or classroom use is granted without fee provided that the copies are not made or distributed for profit or commercial advantage AND that copies 1) bear this notice in full and 2) give the full citation on the first page. It is permissible to abstract these works so long as credit is given. To copy in all other cases or to republish or to post on a server or to redistribute to lists requires specific permission and payment of a fee. Con- be disseminated and used in practice. Knowledge has no real value on its own - it only becomes valuable when people use it make decisions and take action (Sveiby, 1997). However in most disciplines, research findings take a long time to filter into practice, if they ever do at all. There are a number of barriers to the flow of knowledge between research and practice, which originate from both sides of the divide: 
- Practitioner's viewpoint (demand side): The pressures of professional practice leaves little time for practitioners to read journals or attend conferences: time is money, and most organisations do not reward their employees for keeping up with research in their field. Also, the volume of research published every year means that practitioners could not possibly keep up with all the latest research developments in their field —if they did, they would have little time to do anything else.

- Researcher's viewpoint (supply side): Academic research is primarily focused on production rather than distribution of knowledge (Gibbons et al, 1994). Research communities have developed highly efficient mechanisms for transfer of knowledge among themselves (via the processes of publication and citation), but there is little investment in the dissemination of research results into practice. As a result, potentially valuable research ideas are circulated within research communities without ever finding their way into practice. Part of the reason for this is that academic institutions reward researchers for publishing their ideas in scholarly journals and conferences, not for having them applied in practice.

The result of these barriers is under-utilisation of research results and sub-optimal practices. This is undesirable from the point of view of practitioners and researchers:

- Researchers do not get their ideas tested in practice, which is a limiting factor in the development and evolution of these ideas (Wynekoop and Russo, 1997)

- In the absence of relevant knowledge about effectiveness of practices, practitioners persist in using practices that are obsolete or proven not to work. As a result, professional practice has limited ability to learn from its mistakes.

\section{Manufacturing Model of Knowledge Production}

In manufacturing, it is important to pay equal attention to production and distribution. To get maximum value from investments in production of goods, it is necessary to have parallel investments in distribution, to ensure that goods get sold and produce revenue. Similarly, to get maximum value from investments in research, it is necessary to have parallel investments in dissemination of research results, in order to improve practices and achieve social outcomes. The issue of how to transfer research results into practice is rarely addressed by researchers, and requires much more than publication in scholarly journals and conferences, which is normally seen as the endpoint of a research project. However, very little is known about how ideas are diffused in practice. The mechanisms are not as well understood as in the academic world, and rely more on informal channels, such as word of mouth (Gibbons et al, 1994).

\section{Knowledge Management}

Fundamental to understanding the issues involved in transferring research findings to practice is the concept of knowledge management. Knowledge management has only recently emerged as a field of practice in its own right (Sveiby, 1997; Davenport and Prusak, 1998). Knowledge is a high value form of information that can be used to make decisions and take action (Davenport et al, 1998). A key difference between knowledge and information or data is that it is intellectually intensive rather than IT-intensive. Knowledge is the result of human interpretation and analysis rather than data processing. Knowledge can be classified into two broad categories:

- Explicit knowledge: this is knowledge which has been articulated or written down. This is also referred to as "knowledge that", articulated knowledge or theoretical knowledge (Ryle, 1949; Polanyi, 1967; Rescher, 1979; Cohen and Squire, 1980; Cohen, 1984; Sveiby, 1997). 
- Tacit knowledge: this is knowledge which is stored in people's heads. This is also referred to as "knowledge how", embodied knowledge or practical knowledge (Ryle, 1949; Polanyi, 1967; Rescher, 1979; Cohen and Squire, 1980; Cohen, 1984; Sveiby, 1997).

Explicit knowledge is the more familiar form of knowledge, and is found in textbooks, manuals and research articles. However tacit knowledge is more valuable because knowledge must be made tacit to make decisions or to take action. For example, you must read and understand a book (internalise the knowledge or make it tacit) in order to be able to apply the knowledge it contains. However because tacit knowledge is intangible, it is much more difficult to manage. Tacit knowledge can converted into explicit knowledge through the process of externalisation (or codification), and this has been the focus of many knowledge management initiatives in practice (Hansen et al, 1999). However, this is only ever partially successful, as we always know more than we can say_also, some knowledge is not accessible to our consciousness (Polanyi, 1967; Sveiby, 1997).

Formal education focuses on imparting explicit knowledge, while experience in practice supplements this with tacit knowledge through on-the-job learning and skills transfer (Sveiby, 1997). So far, knowledge management has been primarily concerned with managing knowledge flows within single organisations. However the same principles can be applied to the issue of improving knowledge transfers between research and practice within a discipline.

There are two key flows of knowledge which need to occur between research and practice in an applied discipline (Figure 1):

- Practice $\rightarrow$ Research: research activity should be informed by the needs of practice and society. This ensures that research addresses issues that are of social and practical significance (relevance).

- Research $\rightarrow$ Practice: research results should be disseminated and applied in practice. This ensures that research leads to improvements in practices and benefits to society (impact). In any applied discipline, there is an expectation that research will ultimately result in some useful social outcome (Phillips, 1998).

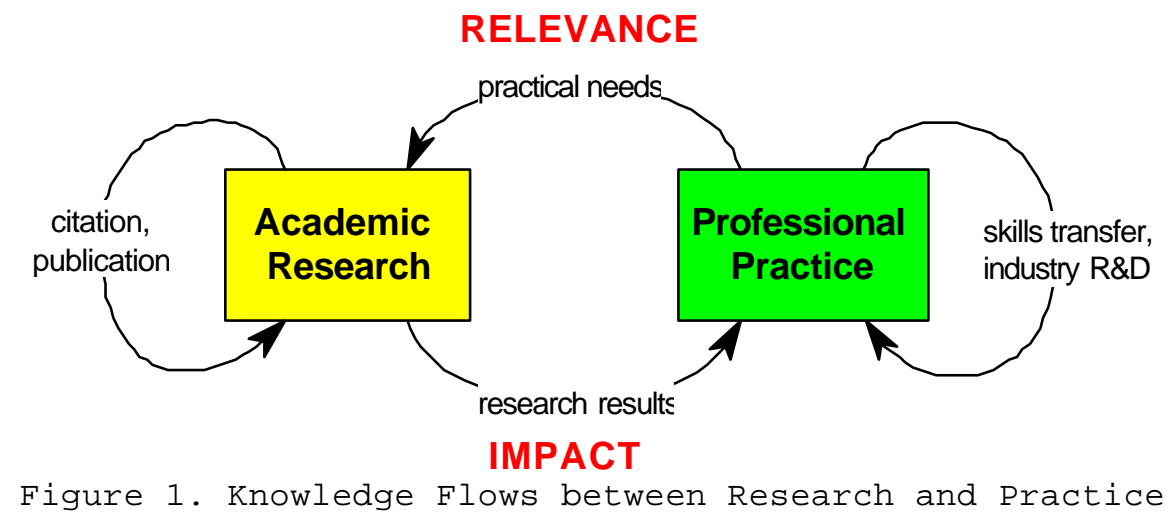

There are also flows of knowledge which occur within each community, although these are not the concern of this paper:

- The processes of citation and publication facilitate knowledge transfers among researchers.

- The process of skills transfer, in which tacit knowledge is transferred via on-the-job learning.

- Industry-based research and development, or "Mode 2" research, in which knowledge is generated and disseminated in the context of practice (Gibbons et al, 1994). This is characteristic of knowledge produced by pharmaceutical companies, consulting companies and IT vendors. 
Improving knowledge transfers between research and practice will result in more effective production and application of research knowledge. In this paper, we focus on the flow of knowledge from research to practice, but this should also help to improve flows of knowledge in the reverse direction, via natural feedback processes.

\section{Current Knowledge Distribution Model}

Until now, there has been an implicit assumption that the best way to transmit research knowledge into practice is to first load it into human minds, via the long and expensive education of professionals (see Figure 2). However there are enormous "voltage drops" along this transmission line (Weed, 1997):

- Only a portion of the available knowledge can be loaded, given the limitations of human information processing and the limited time available for their education.

- Only a portion of the knowledge loaded is retained, and in fact most is quickly forgotten after examinations.

- Only a portion of the knowledge loaded is ever used. It is difficult to predict what knowledge might be useful in the future, so educators try to load as much as possible to prepare for all contingencies. This results in information overload and over-taxing of the retention powers of the human mind.

- Much of the knowledge that is retained quickly becomes obsolete, and there is no assurance that it will be replaced by relevant new knowledge. Once practitioners have completed their formal education, they tend to rely primarily on tacit knowledge acquired through experience in practice— this means they are operating from a knowledge base which is incomplete, out of date and biased.

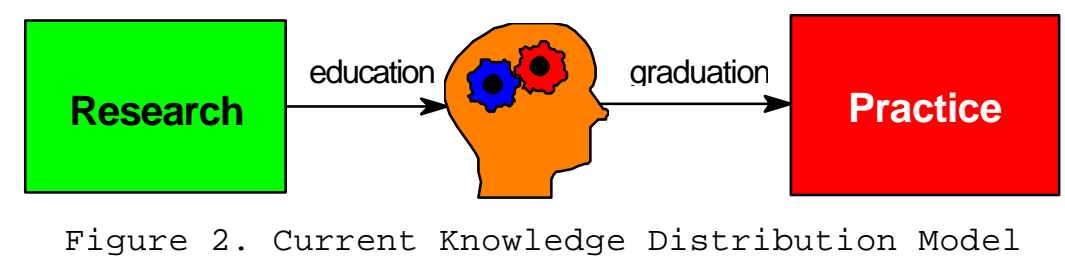

In the "Information Age", this seems a rather archaic and inefficient way of transferring knowledge. Just as science developed the microscope to magnify the power of the human eye, there is a similar need to use information technology to expand the human mind's limited capacity to store and recall large amounts of information (Weed, 1997). Practitioners should not need to load enormous amounts of information into their heads and then try to retrieve it years later (when it is likely to be out of date), but should have the latest research knowledge at their fingertips in the workplace. This will not substitute for the education process, but provides a way of continuously and selectively updating their knowledge. The education process only provides a "snapshot" of knowledge in a field at a point in time. Whenever a practitioner encounters a new situation, they need to be able to update their knowledge on an "as needed" basis — this is the only workable approach given the rate of change in most disciplines.

\section{Objectives of this Paper}

Improving the transfer of knowledge from research to practice requires a channel which connects knowledge producers (researchers) with the intended consumers of this knowledge (practitioners). The World Wide Web provides a global information infrastructure for connecting researchers and practitioners. This paper describes two projects which use the World Wide Web to make research knowledge directly available to practitioners in the workplace. The first is a successful knowledge management project in a health department which provides medical staff with on-line access to the latest medical research at the point of care, in order to improve the qual- 
ity of clinical decision making. The second is a project currently in progress to implement a similar system to support IS decision making. Finally, we draw some general lessons from these projects about transferring knowledge from research and practice, which could be usefully applied in any discipline.

\section{Case Study 1: Evidence-Based Medicine}

\section{Medical Decision Making}

To make appropriate decisions about patient care, medical practitioners need to take into account all relevant medical knowledge and integrate it with detailed data about the patient's condition. Access to the latest medical research can mean the difference between life and death, an accurate or erroneous diagnosis, early intervention or a prolonged and costly stay in hospital (Ayres and Clinton, 1997). However, staying abreast of all the latest developments in medical research is a daunting task - it is estimated that the amount of medical knowledge doubles every five years (Weed, 1997). Faced with information overload, doctors fall back on global judgements based on experience rather than thorough analysis of the relevant medical literature.

For decades, the medical profession has documented high levels of error, far higher than most other industries would tolerate. An adverse event is defined as "an unintended injury or complication which results in disability, death or extended hospital stay, and is caused by health care management rather than the patient's disease" (Wilson et al, 1995). In simple terms, this corresponds to an "error" in medical practice. A study of the Australian health system found that $16.6 \%$ of hospital admissions resulted in adverse events (Wilson et al, 1995). It is estimated that 180,000 people die each year in the United States as a result of adverse events - the equivalent of three jumbo jet crashes every day (Leape, 1994). When the causes of adverse events are investigated, it is found that most (over 80\%) are the result of decision making errors (Wilson et al, 1999).

One of the reasons for the high levels of error in medical practice is its reliance on the unaided human mind (Weed, 1997). Psychological research shows that the human mind is strictly limited in how much information it can memorise and recall (Miller, 1956; Baddeley, 1994; 1999; Eysenck and Keane, 2000). In everyday medical practice, doctors rely heavily on tacit knowledge accumulated as a result of their medical training and subsequent clinical experience. As a result, decisions about the most appropriate treatment for patients are often made based on knowledge that is out-of-date, incomplete and biased. As Weed (1997) says:

"We should never have placed so much power in the hands of those who memorise knowledge, regurgitate it in examinations, forget much of it, specialise in a small piece of it, and never fully integrate what they do with the details of patient's needs."

\section{Evidence-based Medicine}

Medical research findings take a long time to filter into clinical practice (Phillips, 1998). Empirical studies have shown that on average, there is an $8-13$ year time lag (depending on the specialty) between a treatment being proven to work and its adoption in everyday medical practice. It has also been found that $70 \%$ of medical treatments currently in use do not have any reliable evidence to support that they are any more effective than doing nothing (Sackett et al, 1997). The widespread use of disproven or unproven treatments means that the health system is burdened with unnecessary costs and patients are exposed to unnecessary pain, expense, risk and potential adverse side-effects.

Medicine has well-developed mechanisms for transferring tacit knowledge, via the internship process (on the job learning and skills transfer). However for transfer of explicit knowledge, it relies mainly on the formal education process, which as explained previously, is subject to severe "voltage drops". A major barrier to the imple- 
mentation of research findings is the volume and geometric growth of the medical literature. It is not humanly possible to keep up with all the advances in all areas of medical research— the average medical practitioner would have to read 20 articles per day just to keep up with developments in their specialty area (Jordens et al, 1998). It is also difficult for clinicians not trained in research methodology to make sense of the often conflicting research findings in a particular area.

Recognition of such problems led to the concept of evidence-based medicine (EBM). This is an approach that synthesises research findings on the effectiveness of medical treatments to support clinical decision making (Sackett et al, 1997). The purpose of EBM is to ensure that decisions about patient care are based on the latest scientific evidence, where "evidence" is defined as the results of randomised clinical trials (RCT). The aim is to reduce the time lag between the development of new treatments and their use in everyday medical practice, and to avoid the use of unproven or disproven treatments. The goals of EBM can be summarised as follows:

- To use treatments that have been proven to work

- To eliminate the use of treatments that have been proven not to work (disproven treatments)

- To conduct research into treatments whose effects are unknown (unproven treatments)

One of the major methodological tools in EBM is the systematic review, which is a form of meta-analysis of research results (Cochrane, 1972; Glass et al, 1981). Systematic reviews begin with an exhaustive search for published and unpublished research studies addressing a particular medical issue (e.g. treatment of asthma). The next step is to critically evaluate the studies to identify which are of sufficient quality to contribute to decision making. The final step is to pool the results of the studies to arrive at a quantitative estimate of the effectiveness of the treatment(s). Reviews must be regularly updated to take account of new research developments. This reduces the problems of information overload and interpretation of findings faced by medical practitioners, and puts information in a convenient form for decision making in practice.

However synthesising the research evidence is only the starting point for using research to improve practice. Equally important is the dissemination and use of this information. To make a practical difference, systematic reviews must be readily available to medical practitioners, and must be actively used in everyday clinical practice. EBM represents an application of knowledge management in the medical field, although it pre-dates the mainstream knowledge management literature by more than two decades. It focuses on synthesising explicit knowledge in the form of research findings, and disseminating and applying this knowledge in medical practice. It also focuses on generating new knowledge on the effectiveness of treatments.

\section{Organisational Context}

This case study took place in one of Australia's state health departments. The department is one of the largest organisations in Australia, with an annual budget of over AUD\$8 billion and over one hundred thousand staff. Historically, clinical information needs had not been well supported by investments in information technology. The majority of existing information systems supported administrative processes (e.g. financial systems, payroll systems, patient administration systems), with very few systems directly supporting patient care. To address this issue, a committee was formed in September 1995 to specify requirements for medical information at the point of care. It consisted of fifty clinicians from all health disciplines including hospitals, general practice, community health and universities. A proposal was developed for a project called the Clinical Information Access Project (CIAP) to address this need, which was endorsed by senior management in December 1996. The stated objective of the project was: 
"To provide clinicians with access to on-line medical information to support clinical practice, education and research at the point of care".

A pre-implementation survey of 2757 clinicians and medical librarians was carried out to identify the most important knowledge sources to support clinical practice. The system went live on July 4, 1997, taking just over six months from its initial inception to implementation. It has now been in operation for over five years, and is still evolving.

\section{Knowledge Content}

The knowledge content of the system was based on the requirements identified in the pre-implementation survey. It consists of five major components, which correspond to different classifications of knowledge on the tacit/explicit dimension and the internal/external dimension (whether the knowledge was produced inside or outside the organisation), as shown in Table 1. Unlike most knowledge management systems, which primarily focus on internal knowledge (Sveiby, 1997; Davenport et al, 1998; Davenport and Prusak, 1998; Hansen et al, 1999), this system is mainly focused on providing access to external knowledge, and in particular, the results of medical research.

Table 1. Knowledge Components

\begin{tabular}{|l|l|c|}
\cline { 2 - 3 } \multicolumn{1}{c|}{} & \multicolumn{1}{c|}{ EXTERNAL } & INTERNAL \\
\hline \multirow{2}{*}{ EXPLICIT } & $\begin{array}{l}\text { Systematic (EBM) Reviews } \\
\text { On-line Literature Searching } \\
\text { Tools } \\
\text { Pharmaceutical Databases }\end{array}$ & $\begin{array}{c}\text { Clinical Policies and } \\
\text { Protocols }\end{array}$ \\
\hline TACIT & & Listservers \\
\hline
\end{tabular}

\section{Systematic Reviews}

A range of databases are provided that provide on-line access to systematic (EBM) reviews:

- Cochrane Library: this is an electronic database of systematic reviews produced by the Cochrane Collaboration, an international not-for-profit organisation specialising in principles of EBM. The Cochrane Library is recognised as the leading source of EBM reviews.

- APC EBM Reviews: systematic reviews produced by the American College of Physicians.

- Evidence-Based Medicine: systematic reviews produced by the British Medical Association.

- Database of Abstracts of Reviews of Effectiveness: systematic reviews produced by the British National Health Services' Centre for Reviews and Dissemination (NHS CRD).

These databases provide high value knowledge in the form of synthesised research findings. Each EBM review incorporates thousands of hours of critical review and synthesis of research articles by leading medical researchers and practitioners.

\section{On-line Literature Searching Tools}

Access to primary research sources (medical research journals) is also provided: 
- MEDLINE: this is recognised as the leading bibliographic source of medical research, and provides on-line searching with links to full text medical journals.

- CINAHL: provides on-line searching with links to full text nursing and allied health journals.

- PsychINFO: provides on-line searching with links to full text psychiatry and psychology journals

- Healthstar: provides on-line searching with links to full text health administration journals

- Full text journals and textbooks: a range of full text journals and medical textbooks are available on-line.

These databases provide access to the latest medical research findings. Compared to EBM reviews, they have the disadvantage that clinicians must synthesise and interpret the research findings themselves, but have the advantage of broader coverage (EBM reviews are only available for a limited range of topics) and currency (EBM reviews are only updated on an annual basis).

\section{Pharmaceutical Databases}

The system provides a range of on-line sources of drug information:

- MIMS: a comprehensive pharmaceutical database, which includes details of all known drugs, side-effects, interactions and recommended dosages.

- Antibiotic Guidelines: provides decision support in prescribing antibiotics (which are generally poorly prescribed in practice).

- Micromedex: poisons and toxins information.

Up-to-date drug information (e.g. recommended dosage, administration instructions, allergies, side-effects and interactions) is essential for prevention of adverse events. A recent study of adverse events in general practice found that over $50 \%$ were the result of pharmacological errors (Kidd and Veale, 1998). With thousands of drugs currently on the market, and hundreds of new ones released every year, it is impossible for medical practitioners to keep information about all of these in their heads (Milne, 2002).

\section{Clinical Policies and Protocols}

Clinical policies and protocols define standard procedures for handling particular types of cases (e.g. cardiac arrest, road trauma). These play a critical role in medical practice - incorrect protocols or failure to follow protocols represent the most common cause of adverse events (Wilson et al, 1995; Wilson et al, 1999). They represent an important source of explicit knowledge in the organisation. Each hospital defines its own policies and protocols, and there may be wide variations between them based on equipment available and when they were last updated. The system allows clinicians to post their clinical policies and protocols on a voluntary basis for peer review. The purpose of this is to encourage collaboration and sharing of knowledge, and to move towards standardisation and development of "best practices".

\section{Listservers}

Listservers are provided for on-line discussion of problems and issues in particular areas of specialty, for example, asthma, stroke and medical ethics. This facilitates the exchange of ideas and experiences (tacit knowledge) among researchers and clinicians across the organisation. This is especially useful for clinicians in rural and remote areas, who have less opportunity for face-to-face exchange of knowledge. Strictly speaking, it is not possible to transmit tacit knowledge via a listserver - the knowledge must be externalised or codified (transformed into explicit knowledge) before it can be communicated. However listservers provide a more informal and per- 
sonal channel for knowledge transfer that enables communication of knowledge that would normally remain tacit.

\section{Technology Architecture}

The system operates using a single web server located at the organisation's head office, and is available 24 hours a day, 7 days a week. The World Wide Web was chosen instead of intranet technology in order to maximise the reach of the system. Because of the geographic spread of the organisation and the lack of a communications network linking all health care facilities, an intranet would have excluded a large proportion of clinicians. An added advantage of using the World Wide Web is that it allows clinicians to access the system from home, which represents around half the total usage of the system.

\section{Scenario-How the System is used}

To illustrate how the system is used in everyday medical practice, consider the case of a patient who presents with funnel web spider bite. The funnel web spider (Atrax Robustus) is unquestionably the most dangerous spider in Australia. It is a large (6-7 $\mathrm{cm})$, black, aggressive spider, with fangs large and powerful enough to penetrate a fingernail. During a bite, the spider firmly grips its victim and bites repeatedly, and the venom is highly toxic. There is estimated to be 30-40 cases of funnel web spider bite occur each year in Australia. Given the rarity of such cases, it is unlikely that a clinician would be able to rely on previous experience or their medical training to know

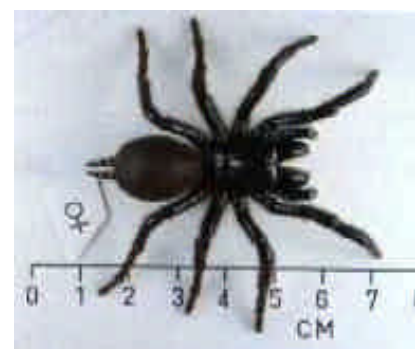

Funnel Web Spider (Atrax Robustus) how to treat such a case. Death occurs between 15 minutes and 3 days following the bite, so prompt action is essential.

From the opening screen, the attending medical officer conducts a search using the phrase "funnel web spider bite" (Figure 3). This initiates a search across all knowledge sources in the system.

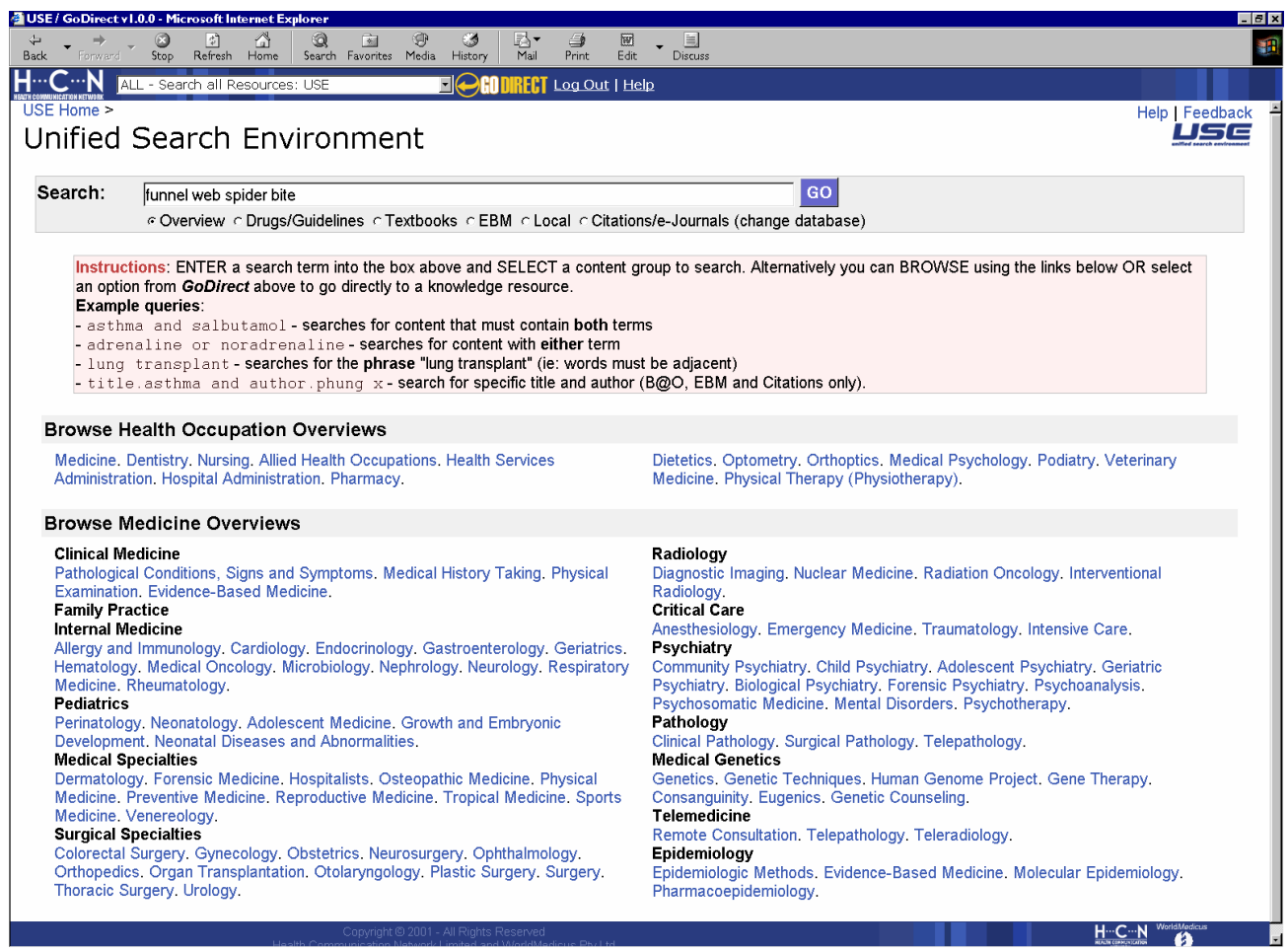

Figure 3. Unified Search Environment 
The top-ranked result of the search is an entry from the Drug and Therapeutic guidelines, which describes the symptoms of funnel web spider bite (for confirming the diagnosis), the antivenom required, recommended dosage, administration instructions, interactions and possible side effects (Figure 4).

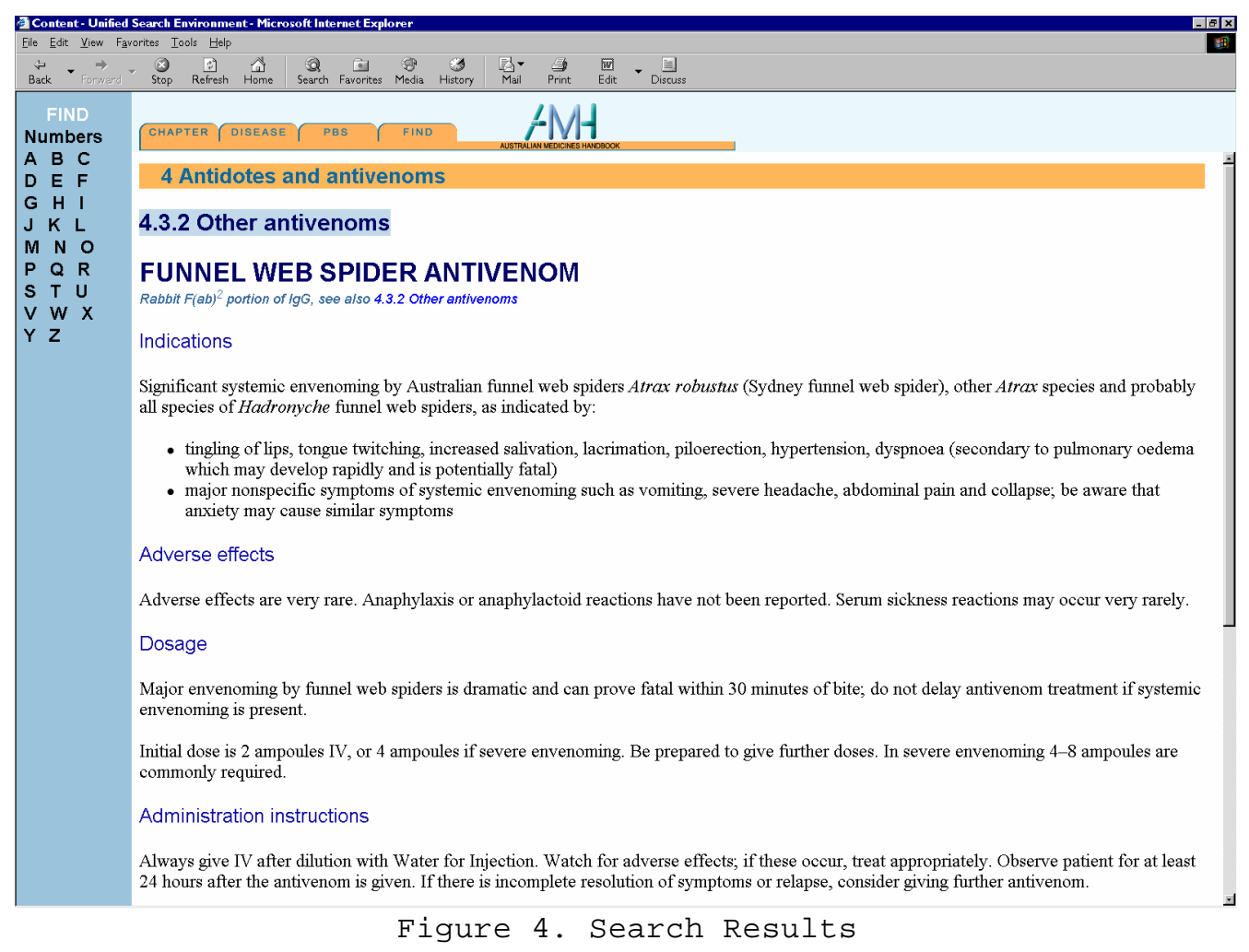

\section{Benefits of the System}

Both anecdotal evidence and perceptions of clinicians support the conclusion that the system has been successful in improving the quality of clinical decision making. A survey of clinicians using the system revealed that over $90 \%$ felt that it had improved patient care. There have also been a number of reported cases where access to the system has saved lives:

- A registrar in a rural hospital was able to save a patient in a critical condition suffering from the Lyssavirus. The Lyssavirus, which is acquired from contact with bats, causes encephalitis in humans and can be fatal if not treated quickly. However because it is a relatively new disease (the first case was discovered in Australia in May 1996), it does not yet appear in medical textbooks. Using MEDLINE, the clinician was able to look up and apply the appropriate treatment.

- Another rural clinician was able to save a patient who was admitted in a critical condition in the middle of the night, suffering from meningitis. The patient did not respond to the normal treatment for this condition, indicated by the standard clinical protocol. A MEDLINE search revealed a new drug treatment that had immediate results.

- Finally, a clinician was able to save a child who had been bitten by a funnel web spider by looking up the anti-venom in Micromedex. Funnel web spider bite can be fatal, especially in children, and for the antivenom to be effective, it must be applied within 30 minutes. Having on-line access to this information almost certainly meant the difference between life and death. 


\section{Towards Evidence-Based Medical Practice}

This system provides a mechanism for moving away from experience based medical practice (based on tacit knowledge) and towards evidence-based medical practice (based on explicit knowledge in the form of medical research). It provides a way of putting the theory of evidence-based medicine into practice, and using information technology to supplement the limited capability of the human mind to store and recall information. The system provides a direct channel for transfer of research results to clinical practice, and also directly connects researchers and practitioners via listservers. While the concept of EBM has been around for over three decades, the use of IT to provide on-line access to medical evidence in the workplace operationalises the concept in clinical practice.

Prior to this system, clinicians only had access to medical research via libraries attached to the larger hospitals. However these were generally not open after hours or on weekends and many smaller hospitals did not have libraries at all. Also, because of the pressures of clinical practice, medical practitioners rarely had the time to visit the library during working hours. Using this system, clinicians have on-line access to the latest medical research 24 hours a day, 7 days a week, both in the workplace and at home.

One of the explanations for the success of this system and its acceptance by clinicians is that unlike traditional decision support systems (DSS), which have proven to be largely unsuccessful in medical practice, it does not tell the clinician what to do. Twenty years of DSS research has revealed a persistent problem, in that decision makers either ignore or modify the advice given by the DSS (Lim and O'Connor, 1995; Turban, 1995; Lawrence and Sim, 1999; Goodwin, 2000). A range of studies in different environments have shown decision makers tend to trust their own experience and judgement much more than the DSS, even when presented with overwhelming evidence that they would perform much better by simply following the system's advice. A possible explanation for this is that people, and especially highly educated and experienced professionals, do not like being told what to do by a computer. The system described in this study supports human decision making by providing information about available treatments and the evidence for and against them. However it leaves the final decision about what to do to the clinician—it therefore empowers human judgement and allows them to incorporate their experience and judgement into the decision making process.

\section{Removing the Knowledge Gap between Practitioners and Consumers}

If use of the system was extended to consumers, it would potentially have an even greater impact. In particular, patients could use it to look up the latest evidence about their own medical conditions. If they found that there was no reliable evidence to support use of the treatment they were being given, or that there was a better, clinically proven treatment available, they would rightly question their doctor's decision. The medical profession currently enjoys almost "god like" status in society, as a result of the wide knowledge gulf between medical practitioners and consumers. Patients are often uninformed, passive objects in the medical care process, and rarely question their doctor's advice (Weed, 1997). Making the latest research evidence available to patients would help to reduce the knowledge gulf between doctors and patients. While such a move might be resisted by medical practitioners - as knowledge is power and most professional groups are concerned with maintaining their power and status - it would help to improve accountability and the quality of health care. 


\section{Case Study 2: Evidence-based IS Practice}

\section{Decision Making in IS Practice}

There is an enormous amount of IS research published each year, but very little of this knowledge finds its way into practice (Bubenko, 1986; Keen, 1991; Galliers, 1994; Benbasat and Zmud, 1999; Davenport and Markus, 1999; Moody, 2000). IS practitioners rarely refer to research evidence to make decisions but rely instead on their own experience, talking to peers, articles in the popular press or advice from consultants and vendors ("expert opinion"). However all of these knowledge sources are highly subjective and anecdotal. This means that, like medical practitioners, but to a much greater extent, they are operating from an incomplete and biased knowledge base.

Like medical practice, IS practice also exhibits high levels of errors. For example, empirical studies show that only $16.2 \%$ of software projects are completed on-time and on-budget (Standish Group, 1995). This represents a success rate about the same as the error rate in medical practice (cf. 16.6\% of hospital admissions result in adverse events). Part of the reason for the high error rates in IS practice is the failure to learn from mistakes and the persistent use of practices which do not work. Research plays a vital role in enabling a profession to learn from its mistakes and in evaluating the effectiveness of practices.

The major reasons why IS practitioners do not refer to published research are:

- Time pressures: The constant deadline pressure and long working hours in IS practice leaves little or no time to read journals or attend conferences.

- Information overload: The volume of IS research published means it is not humanly possible for IS practitioners (and challenging even for full-time researchers) to keep up with all the relevant research in their area of practice. A recent study identified over 233 journals in the IS field (Hardgrave and Walstrom, 1997). The result is a situation of massive information overload for IS practitioners.

- Accessibility: the average IS practitioner would have great difficulty understanding papers published in the leading IS journals, because of the excessive focus on rigour and use of complex statistics and mathematics. IS research papers are primarily written for other researchers (conference and journal reviewers), which acts as a barrier to utilisation of research findings in practice (Moody, 2000).

A number of industry research organisations have emerged to support IT decision making in practice. However while this research is more accessible and relevant to practitioners, it is of questionable validity.

\section{Towards Evidence-based IS Practice}

The concept of evidence-based medicine - of making decisions based on the latest research evidence rather than on experience and opinions - is something that has clear application for the IS field. The IS field exhibits far more serious problems than medicine in transferring knowledge from research to practice, and the principles of EBM would help to bring research and practice closer together. There are important similarities between IS and medicine, which suggest that the concept can be adapted from one field to another. Both are applied rather than pure disciplines, which focus on applying technology to solve practical problems (Moody, 2000). While medical practitioners apply medical interventions to improve the health of their patients, IS practitioners apply IT-based interventions to improve organisational effectiveness. The problem of transferring research results to practice is essentially the same in both disciplines, though in IS the problem is much worse.

By analogy to evidence-based medicine, the objectives of evidence-based IS practice would be to: 
- Use practices that have been proven to work

- Eliminate practices that do not work (disproven practices)

- Conduct research into practices whose effectiveness is unknown (unproven practices)

The third objective would provide direction for IS research in evaluating the effectiveness of practice, which would help to improve its relevance (the feedback loop in Figure 1).

The system described in the first case study provides a successful solution to the problem of transferring research knowledge to practice. It operationalises the concept of EBM in the form of a web-based information system. In this case study, we adapt this solution to the problem of transferring knowledge from IS research to IS practice. This is an example of analogical reasoning, a problem solving approach in which a solution is found to a problem by looking at how a similar problem has been successfully solved in another (referent) domain (see Figure 5). (Gentner, 1983; Holyoak, 1985; Keane, 1985; 1988). In this case:

- The target domain is IS, while the referent domain is medicine;

- The problem to be solved is that of transferring IS research knowledge to IS practice, while the exemplar problem is the transfer of medical research knowledge to medical practice;

- The exemplar solution is the webbased system described in the first case study, while the target solution is a similar system to support evidence-based IS practice.

Analogical reasoning represents a particularly useful research approach for an immature discipline like IS, and a way

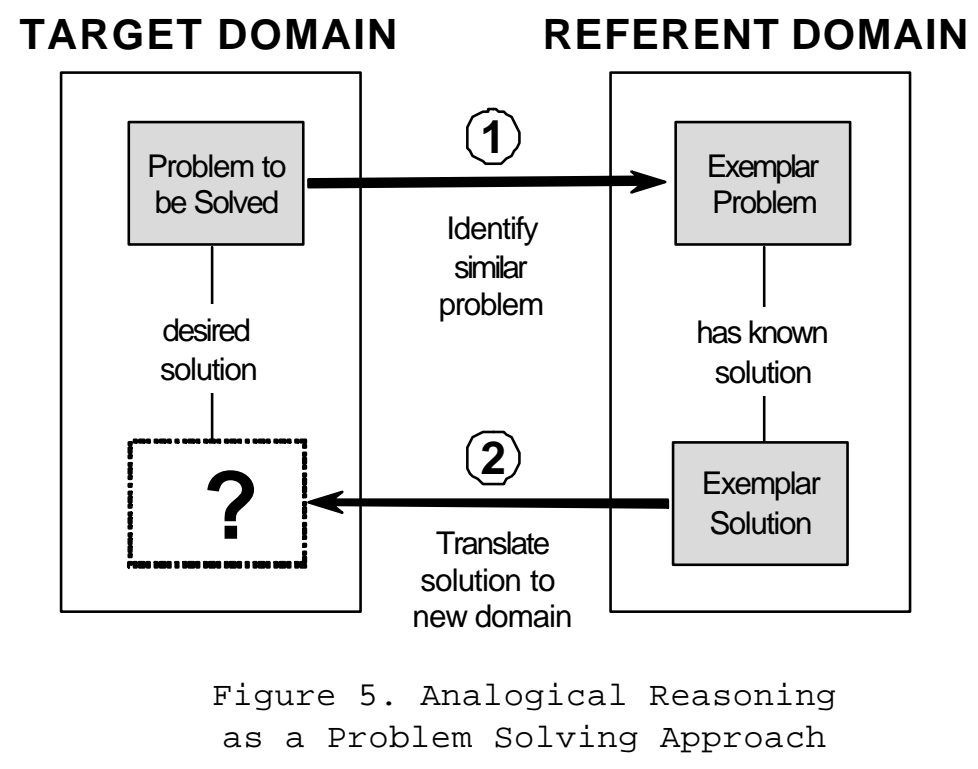
of learning from other, more established disciplines (in this case, medicine).

\section{Project Objectives}

The objective of this project is to develop a web-based system to provide IS practitioners with access to the latest IS research, to support decision making in the workplace. This is an ambitious project, which attempts to build a comprehensive knowledge base to support IS practice. This is an application of principles of knowledge management on a large scale - the level of an entire discipline — and is a joint venture between a research institution (Monash University) and the association for IS professionals in Australia (the Australian Computer Society).

The design of the system will be closely based on the system described in the first case study. The system will incorporate the following components:

- On-line literature searching: this will provide sophisticated searching of IS journals with links to full text journals. This facilitates the transfer of explicit knowledge between research and practice. A number of existing 
on-line literature search engines could be used for this purpose. While such search engines are standard "tools of the trade" for IS researchers, few practitioners have access to them in the workplace.

- Systematic reviews: this will involve the formation of expert committees, consisting of both researchers and practitioners, to review research findings and synthesise results on particular topics. This is a value-added process which will put research results in a highly synthesised form to support decision making in practice. This is a process of synthesising explicit knowledge and provides a way of making research results more accessible to practitioners and reducing problems of information overload (Atkins and Louw, 2000; Moody, 2000).

- Posting of policies, standards, architectures, software evaluations etc. by practitioners for peer review: this would be done by subscribers on a voluntary basis to facilitate knowledge sharing, development of "best practices" and industry standardisation. This facilitates sharing of explicit knowledge among practitioners. Commercial interests will be a limiting factor in such knowledge sharing, as many organisations will want to protect their intellectual property and competitive interests, but in many areas, there will be clear mutual benefits in sharing knowledge.

- Listservers to promote discussion on particular issues: this would facilitate transfer of tacit knowledge in the form of ideas and experiences between practitioners and researchers.

The intention is to make the system available free of charge to all members of the Australian Computer Society.

\section{Potential Benefits}

Implementation of this system will help to:

- Improve the dissemination and application of IS research results in practice

- Reduce time lag between development of new research knowledge and its application in practice

- Help to reduce the use of practices that don't work and thereby facilitate learning from mistakes

- Connect researchers and practitioners together (via listservers)

- Provide a comprehensive and up-to-date knowledge base to support decision making in IS practice

- Improve the professionalism of IS practice, by moving towards evidence-based rather than opinion and experience based practice

- Reduce problems of information overload for IS practitioners and make it easier for them to stay abreast of the latest research (via systematic reviews)

- Improve sharing of knowledge among practitioners (via posting of policies, standards etc.)

As in the previous study, this system would also be beneficial to consumers of IS services. Many end users are relatively uninformed and rely heavily on advice from vendors and consultants which is often biased (and sometimes quite predatory). Consultancy firms and IT vendors are notorious for taking advantage of users' lack of knowledge to sell them products or services that they don't need or are not effective. Making the latest IS research available to consumers of IS products and services will help to ensure that they get better value for money and also improve accountability of the IS profession.

\section{Potential Barriers}

There are also a number of potential barriers to successful implementation of this system, which reflect some fundamental differences between medical and IS practice: 
- Scepticism about IS research: IS practitioners tend to be rather sceptical of the value of academic research. This would be a significant barrier to adoption and use of the system. However if the system is found to be useful in practice, and a critical mass of practitioners start using it, normative influences are likely to overcome this scepticism (Green, 1998).

- Lack of evidence: in most areas of IS practice, there isn't a sufficient body of research to conduct effective systematic reviews. Because of the rate of technological change, it is difficult for IS research to keep pace with new technological developments and evaluate them in a timely manner. In addition, the conclusions from IS research studies tend to be much weaker than those in medicine. Because most IS interventions are applied at the organisational level, randomised clinical trials, which are the standard method for obtaining evidence in medicine, are not as widely applicable — in fact, some researchers have argued that experimental methods are inappropriate in IS research. IS research relies much more heavily on qualitative research methods, which leads to less definite conclusions about effectiveness of practices.

- Relevance of IS research: A number of authors have criticised IS research for not addressing questions that are relevant to practice (Keen, 1991; Galliers, 1994; Benbasat and Zmud, 1999; Davenport and Markus, 1999). This is a "supply side" issue that is difficult to address in the short term. However as research results are more widely disseminated in practice, relevance of research should be improved through natural feedback mechanisms. In addition, listservers provide a direct channel for practitioners to influence research priorities.

- Need to incorporate non-academic research sources: Gibbons et al (1994) argue that universities no longer have a monopoly on knowledge production, and that especially in high tech fields, most new knowledge originates from practice than from academic research. This suggests that it is important to include industry based knowledge sources rather than restricting knowledge sources to academic research.

\section{Conclusion}

\section{Summary of Findings}

For research to make a practical difference, research results must be readily available to practitioners, and must be actively used and implemented in everyday practice (Jordens et al, 1998; Phillips, 1998). The World Wide Web provides an infrastructure for connecting the producers of research knowledge (researchers) with the intended consumers of this knowledge (practitioners). This paper has described a successful system which provides on-line access to the latest medical research to support clinical decision making at the point of care. It also describes a project currently in progress to develop a similar system to support IS practice. Neither of these projects would have been possible prior to the development of the World Wide Web, and illustrate how it has the potential to improve practices and also to allow consumers to become more informed. Providing this information to consumers will erode the power base of practitioners, which they are likely to resist, but ultimately it is good for society and will be a powerful impetus towards improving practices.

\section{Towards a Paradigm for Evidence-based Practice}

The systems movement was founded with the objective to "investigate the similarities of concepts, laws and models from various fields and to help in useful transfers from one field to another" (von Bertalanffy, 1968). Systems theory uses the process of generalisation as an approach to achieving such transfers. A problem in a particular domain can be generalised to a systems problem by removing all domain specific aspects (Klir, 1985). The solution to the systems problem can then be applied in a wide range of domains. 
Evidence-based medicine (EBM) is an approach for improving the effectiveness of medical practice. More generally, it provides a conceptual framework for improving knowledge transfers from research to practice. The problems addressed by EBM are experienced to a greater or lesser extent in all applied disciplines, and similar principles could be applied in any field to improve knowledge transfers from research to practice. The principles of EBM can be generalised to the systems level to develop a general paradigm for evidence-based practice, which can then be applied in multiple domains (Figure 6).

The two case studies described in this paper represent practical solutions to the problem of transferring knowledge from research to practice, which operationalise the concept of evidence-based practice in the form of information systems. The key elements of these systems are:

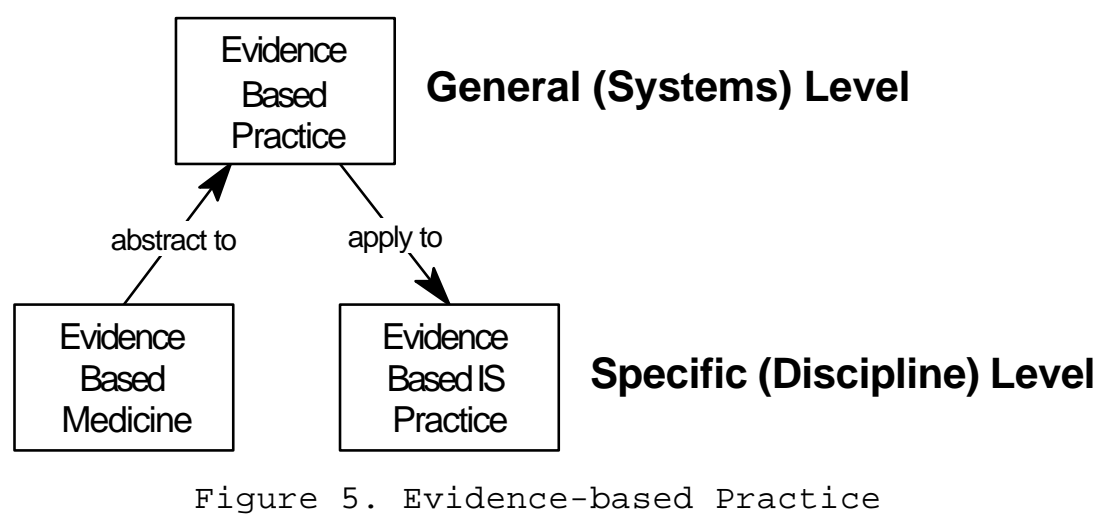

- Systematic reviews: synthesis of research literature to increase accessibility to practitioners and reduce time required to find and evaluate evidence.

- On-line literature searching tools: access to primary research sources with value-added search capabilities

- Posting of practitioner-developed "knowledge products" (e.g. policies, standards, procedures): sharing of explicit knowledge to support development of "best practices" and achieving industry standardisation.

- Listservers: informal channels for sharing knowledge between researchers and practitioners.

These systems demonstrate how information technology can be used to expand the human mind's limited capacity to store and recall large amounts of information. Providing practitioners with access to the latest research in the workplace provides a form of "just-in-time" learning, which allows them to selectively update their knowledge on an "as needed" basis. This will help to supplement the education process, which provides a "snapshot" of knowledge in a field at a point in time.

\section{References}

Atkins, C. \& Louw, G. (2000). Reclaiming knowledge: The case for evidence based information systems . Proceedings of the 8th European Conference on Information System (ECIS2000). Vienna, Austria.

Ayres, D.H.M. \& Clinton, S. (1997). The user connection: Making the clinical information systems vision work in NSW health. Health Informatics Conference (HIC '97). Sydney, Australia.

Baddeley, A.D. (1994). The magical number seven: Still magic after all these years? Psychological Review, 101, 2.

Baddeley, A.D. (1999). Essentials of human memory. Hove, England: Psychology Press.

Benbasat, I. \& Zmud, R.W. (1999). Empirical research in information systems: The practice of relevance. MIS Quarterly, 23, 1, March.

Bubenko, J.A. (1986). Information systems methodologies - A research view. Information Systems Design Methodologies: Improving the Practice, T.W. Olle, H.G. Sol and A.A. Verrijn-Stuart, (Eds.), North-Holland.

Cochrane, A.L. (1972). Effectiveness and efficiency: Random reflections on health services. London: Royal Society of Medicine Press. 
Cohen, N.J. (1984). Preserved learning capacity in amnesia: Evidence for multiple memory systems . In L. Squire \& N. Butters (Eds.), Neuropsychology of Memory. New York: Guilford Press.

Cohen, N.J. \& Squire, L.R. (1980). Preserved learning and retention of pattern analysing skill in amnesia using perceptual learning. Cortex, 17, pp. 273-278.

Davenport, T.H., De Long, D.W. \& Beers, M.C. (1998). Successful knowledge management projects. Sloan Management Review, 39, 2, pp. 43-52.

Davenport, T.H. \& Markus, M.L. (1999). Rigour vs. relevance revisited: Response to Benbasat and Zmud. MIS Quarterly, 23, 1, March.

Davenport, T.H. \& Prusak, L. (1998). Working knowledge: How organisations manage what they know. Boston, MA: Harvard Business School Press.

Eysenck, M.W. \& Keane, M.T. (2000). Cognitive psychology: A student's handbook. Hove, England: Lawrence Erlbaum

Galliers, R.D. (1994). Relevance and rigour in information systems research: Some personal reflections on issues facing the information systems research community. Proceedings of the IFIP TC8 Conference on Business Process Reengineering: Information Systems and Challenges. Gold Coast, Australia.

Gentner, D. (1983). Structure-mapping: A theoretical framework for analogy. Cognitive Science, 7.

Gibbons, M., Limoges, C., Nowotny, H., Schwartzman, S., Scott, P. \& Trow, M. (1994). The new production of knowledge: The dynamics of science and research in contemporary societies. Sage Publications.

Glass, G.V., McGaw, B. \& Smith, M.L. (1981). Meta analysis in social research. London: Sage.

Goodwin, P. (2000). Improving the voluntary integration of statistical forecasts and judgment. International Journal of Forecasting, 16, pp. 85-99.

Green, C. (1998). Normative influence on the acceptance of information technology. Small Group Research, 29, 1, February.

Hansen, M., Nohria, H. \& Tierney, T. (1999). What's your strategy for managing knowledge? Harvard Business Review, March/April.

Hardgrave, B.C. \& Walstrom, K.A. (1997). Forums for MIS scholars. Communications of the ACM, 40, 11, November.

Holyoak, K.J. (1985). The pragmatics of analogical transfer. The Psychology Of Learning And Motivation, 19.

Jordens, C.F.C., Hawe, P., Irwig, L.M., Henderson-Smart, D.J., Ryan, M., Donoghue, D.A., Gabb, R.G. \& Fraser, I.S. (1998). Use of systematic review of randomised trials by Australian neonatologists and obstetricians. Medical Journal of Australia, March 16.

Keane, M. (1985). On drawing analogies when solving problems : A theory and test of solution generation in an analogical problem solving task. British Journal Of Psychology, 76.

Keane, M. (1988). Analogical problem solving. New York:Wiley.

Keen, P.G.W. (1991). Relevance and rigour in information systems research: Improving quality, confidence, cohesion and impact. In H.-E. Nissen, H.K. Klein \& R. Hirschheim, (Eds.), Information Systems Research: Contemporary Approaches and Emergent Traditions. North Holland: Elsevier Science.

Kidd, M.R. \& Veale, B.M. (1998). How safe is Australian general practice and how can it be made safer? Medical Journal of Australia, July 20.

Klir, G.J. (1985). Architecture of systems problem solving. New York: Plenum Press.

Lawrence, M.J. and SIM, W. (1999). Prototyping a financial DSS. Omega, 27, 4, pp. 445-450.

Leape, L. (1994). Error in medicine. Journal of the American Medical Association (JAMA), 272.

Lim, J. \& O'Connor, M. (1995). Judgemental adjustment of initial forecasts: Its effectiveness and biases. Journal of Behavioural Decision Making, 8, pp. 149-168.

Miller, G.A. (1956). The magical number seven, plus or minus two: Some limits on our capacity for processing information. The Psychological Review, March.

Milne, E.B.G.W.A. (2002). Drugs: Synonyms \& properties (2nd ed.). Burlington, VT: Ashgate. 
Using the WWW to Connect Research and Practice

Moody, D.L. (2000). Building links between IS research and professional practice: Improving the relevance and impact of IS research. In R.A. Weber \& B. Glasson, (Eds.), International Conference on Information Systems (ICIS'00), Brisbane, Australia, December 11-13.

Phillips, P.A. (1998). Disseminating and applying the best evidence. Medical Journal of Australia, March.

Polanyi, M. (1967). The tacit dimension. London: Routledge \& Kegan-Paul.

Rescher, N. (1979). Cognitive systematization. Oxford: Basil Blackwell.

Ryle, G. (1949). The concept of mind. Chicago: University of Chicago Press.

Sackett, D.L., Richardson, W.S., Rosenberg, W. \& Haynes, R.B. (1997). Evidence based medicine: How to practice and teach EBM. New York: Churchill Livingstone.

Standish Group (1995). The CHAOS report into project failure. The Standish Group International Inc. Available on-line at http://www.standishgroup.com/visitor/chaos.htm

Sveiby, K.-E. (1997). The New organisational wealth: Managing and measuring knowledge-based assets. San Francisco: Berret-Koehler.

Turban, E. (1995). Decision support and expert systems: Management support systems, (4th ed.). Engelwood Cliffs, NJ: Prentice Hall.

Von Bertalanffy, L. (1968). General systems theory: Foundations, development, applications. Braziller.

Weed, L.L. (1997). New connections between medical knowledge and patient care. British Medical Journal, 315, July 26.

Wilson, R.M., Harrison, B.T., Gibberd, R.W. and Hamilton, J.D. (1999): "An analysis of the causes of adverse events from the quality in Australian health care study", The Medical Journal of Australia, May 3.

Wilson, R.M., Runciman, W.B., Gibberd, R.W., Harrison, B.T., Newby, L. \& Hamilton, J.D. (1995). The quality in Australian health care study. The Medical Journal of Australia, November 6.

Wynekoop, J.L. \& Russo, N.L. (1997). Studying systems development methodologies: An examination of research methods. Information Systems Journal, 7, 1, January.

\section{Biography}

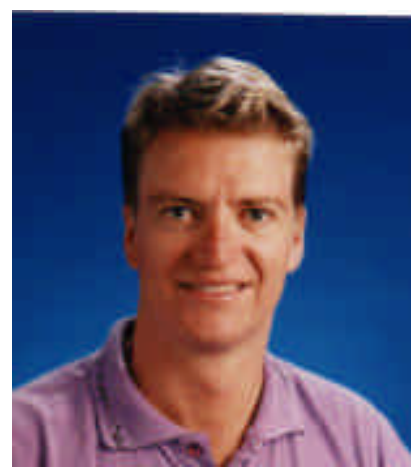

Daniel Moody is a Visiting Professor in the Department of Software Engineering, Charles University Prague (visiting from the School of Business Systems, Monash University, Australia). He has a PhD in Information Systems from the University of Melbourne and has held academic positions at the Universidad de Valencia, the Norwegian University of Science and Technology (NTNU), the University of Melbourne, the University of New South Wales, Queensland University of Technology and the University of Queensland. He is the current Australian President of the Data Management Association (DAMA) and Australian World-Wide Representative for the Information Resource Management Association (IRMA). Daniel has held senior IT management positions in some of Australia's largest commercial organisations, and has consulted to a wide range of organisations both in Australia and overseas. His research interests include data modelling, information resource management, information economics, data warehousing, IT education, health informatics and knowledge management. He has published over 60 papers in the IS field, in both practitioner and academic forums, and has chaired a number of national and international conferences. 